\title{
MULTIDIMENSIONAL DUSTY GASDYNAMICAL MODELS OF INNER COMETARY ATMOSPHERES
}

\author{
TAMAS I. GOMBOSI \\ Space Physics Research Laboratory \\ Department of Atmospheric, Oceanic and Space Sciences \\ The University of Michigan \\ Ann Arbor, MI 48109 \\ USA
}

ABSTRACT. The presently available multidimensional dusty gasdynamic cometary atmosphere models are reviewed. This paper examines the simplifying assumptions and their potential consequences. Special attention is paid to the question of potential nightside dust accumulation presently predicted by Körösmezey and Gombosi (1989).

\section{Introduction}

It was recognized as early as the mid-1930s that gas outflow plays an important role in cometary dust production. Present models use Probstein's two-component approach, which applies hydrodynamic conservation equations for the gas component, neglects the random motion of the dust particles and uses free molecular approximation to describe the gas-dust interaction (Probstein, 1968). In this dusty gas dynamic treatment, the traditional gas energy conservation equation is replaced by a combined dust-gas energy integral.

The comet Halley imaging experiments showed that cometary activity is concentrated to limited areas on the sunlit side of the nucleus, with most of the dust ejection coming from fairly localized jets (Keller et al., 1987). These results underscored the necessity for multidimensional dusty gas flow models, which can describe the highly anisotropic gas and dust flows in the inner cometary coma. The first multidimensional dusty gas model was published by Kitamura (1986), who developed a time-dependent 2D dusty gasdynamic code using one characteristic dust size, simple energetics, and a highly simplified chemistry. In a follow-up paper, Kitamura (1987) also investigated the multidimensional dynamics of isolated jets (when all the gas and dust is produced by a limited active region), as well as the viscous flow of pure gas jets. In the isolated jet calculation (Kitamura, 1987), no background gas ejection was assumed, and therefore the nightside gas became too tenuous to be described by inviscid hydrodynamic equations. Based on this consideration, this model did not solve the gas and dust transport equations beyond the terminator in the case of isolated dusty jets. In his two papers, Kitamura $(1986,1987)$ concentrated on 
the study of steady-state solutions because he thought they were the first important step toward a better understanding of cometary jet related phenomena. However, in the light of the latest Giotto imaging results, it seems to be important to extend the models to the nightside as well.

Recently, Gombosi and Körösmezey developed a new time-dependent 2D dusty gas dynamics model of the inner cometary atmosphere (Gombosi and Körösmezey, 1988, 1989; Körösmezey and Gombosi, 1989). Their model considers a single characteristic dust size $(0.65 \mu \mathrm{m})$ and solves the inviscid flow equations for an expanding water vapor atmosphere. The model uses simplified photochemistry and approximates the radiation field by the unattenuated solar radiation. These authors applied a new second-orderaccurate Godunov-type numerical scheme with dimensional splitting (cf. van Albada et al., 1982), which is especially suited to treat shocks and other fast transients.

\section{Model}

\subsection{GOVERNING EQUATIONS}

The physical model used by the multidimensional dusty gas dynamic calculations (Kitamura, 1986, 1987; Körösmezey and Gombosi, 1989) describes an interacting mixture of expanding cometary gas and non-volatile dust grains. The gas component is assumed to be a non-viscid, compressible perfect fluid, while the gas-dust interaction is described using the free molecular approximation. The free-molecular assumption (that the mean free path of molecular collisions is much larger than the grain size) is easily satisfied at most comets. In a reasonably good approximation, the mean free path of molecular collisions is $\lambda \approx 2 \times 10^{-4} / \mathrm{z}$, where $\lambda$ is measured in units of centimeters, and the cometary gas mass production rate, $\mathrm{z}$, is given in units of $\mathrm{g} \cdot \mathrm{cm}^{-2} \cdot \mathrm{s}^{-1}$. At the active regions of comet Halley, $\mathrm{z} \approx 10^{-5} \mathrm{~g} \cdot \mathrm{cm}^{-2} \cdot \mathrm{s}^{-1}$, so $\lambda \approx 1 \mathrm{~m}$. The mean free path at the surface of the much less active comet Giacobini-Zinner was approximately $10 \mathrm{~m}$, which is still small compared with the characteristic scale of the flow. The fluid approximation essentially loses validity when one considers surface regions with negligible gas production.

As the vaporized gases leave the surface, they drag away some of those dust grains that have already been evacuated of their ice component. The gas drag force accelerates the dust particles to terminal velocities that become comparable with the gas flow velocity for the smaller grains. The mass, momentum and energy conservation equations of the singlefluid, inviscid, perfect gas are the following:

$$
\begin{aligned}
& \frac{\partial \rho}{\partial t}+(\mathbf{u} \cdot \nabla) \rho+\rho(\nabla \cdot \mathbf{u})=0 \\
& \rho \frac{\partial \mathbf{u}}{\partial t}+\rho(\mathbf{u} \cdot \nabla) \mathbf{u}+\nabla p=-\mathbf{F}_{g d} \\
& \frac{1}{\gamma-1} \frac{\partial p}{\partial t}+\frac{1}{\gamma-1}\left(\mathbf{u}^{\prime} \cdot \nabla\right) p+\frac{\gamma}{\gamma-1} p(\nabla \cdot \mathbf{u})=Q_{\text {ext }}-Q_{g d}
\end{aligned}
$$


where $\mathrm{t}=$ time, $\rho=$ gas mass density, $\mathrm{p}=$ gas pressure, $\mathbf{u}=$ gas velocity, $\mathbf{F}_{\mathrm{gd}}=$ gasto-dust momentum transfer rate, $\gamma=$ gas specific heat ratio (in the present models, a $\gamma=4 / 3$ value is used, characterizing $\mathrm{H}_{2} \mathrm{O}$ molecules), $\mathrm{Q}_{\mathrm{gd}}=$ gas-to-dust energy transfer rate, and $\mathrm{Q}_{\mathrm{ext}}=$ external heating rate. Both axisymmetric models assume that photodissociation of water molecules represents the major external energy source in the innermost coma, and they neglect the radiative cooling/heating effects (Kitamura, 1986, 1987; Körösmezey and Gombosi, 1989).

It is assumed that dust particles do not undergo any further sublimation or fragmentation in the coma (there is recent indication that this assumption is probably violated); consequently, the dust size distribution function, $\mathrm{f}_{\mathrm{a}}$, must obey the following continuity equation:

$$
\frac{\partial \mathrm{f}_{\mathrm{a}}}{\partial \mathrm{t}}+\left(\mathbf{V}_{\mathrm{a}} \cdot \nabla\right) \mathrm{f}_{\mathrm{a}}+\mathrm{f}_{\mathrm{a}}\left(\nabla \cdot \mathbf{V}_{\mathrm{a}}\right)=0
$$

. where $\mathbf{V}_{\mathrm{a}}=$ dust particle velocity and $\mathrm{a}=$ dust radius. In the innermost coma, where most of the gas-dust interaction takes place, the radiation pressure and gravitational effects can be neglected, and the dust momentum equation becomes:

$$
\frac{\partial \mathbf{V}_{\mathrm{a}}}{\partial t}+\left(\mathbf{V}_{\mathrm{a}} \cdot \nabla\right) \mathbf{V}_{\mathrm{a}}=\frac{3}{4 \mathrm{a}_{\mathrm{a}}} \mathrm{pC}_{\mathrm{D}}^{\prime} \mathbf{s}_{\mathrm{a}}
$$

where $\rho_{\mathfrak{a}}=$ dust bulk density. The dimensionless gas-dust relative velocity, $\mathbf{s}_{\mathbf{a}}$, is defined as

$$
\mathbf{s}_{\mathrm{a}}=\frac{\mathbf{u}-\mathrm{v}_{\mathrm{a}}}{\sqrt{2 \frac{\mathrm{k}}{\mathrm{m}} \mathrm{T}}}
$$

where $\mathrm{k}=$ Boltzmann's constant, $\mathrm{m}=$ mass of gas molecules, and $\mathrm{T}=$ gas temperature . The modified free molecular drag coefficient, $C_{D}^{\prime}=s_{a} C_{D}$ (where $C_{D}$ is the free molecular drag coefficient for a perfect sphere), is given in Kitamura (1986) and Körösmezey and Gombosi (1989). In the presence of an external radiation field, the energy balance equation for a single dust particle is (cf. Kitamura, 1986; Körösmezey and Gombosi, 1989):

$$
\mathrm{C}_{\mathrm{a}}\left[\frac{\partial \mathrm{T}_{\mathrm{a}}}{\partial \mathrm{t}}+\left(\mathrm{V}_{\mathrm{a}} \cdot \nabla\right) \mathrm{T}_{\mathrm{a}}\right]=\frac{3}{\mathrm{a} \rho_{\mathrm{a}}}\left[\mathrm{pC}_{\mathrm{H}} \sqrt{\mathrm{T}}+\frac{1-\mathrm{A}_{\mathrm{vis}}}{4} \mathrm{I}_{\mathrm{rad}}-\left(1-\mathrm{A}_{\mathrm{IR}}\right) \sigma \mathrm{T}_{\mathrm{a}}^{4}\right]
$$

where $\mathrm{T}_{\mathrm{a}}=$ dust grain temperature, $\mathrm{C}_{\mathrm{a}}=$ dust specific heat, $\mathrm{A}_{\mathrm{vis}}$ and $\mathrm{A}_{\mathrm{IR}}$ are the visible and infrared dust albedos, respectively, $\sigma=$ Stefan-Boltzmann constant, and $\mathrm{I}_{\mathrm{rad}}=$ radiation energy flux reaching the dust particle. The modified thermal accommodation coefficient, $\mathrm{C}_{\mathrm{H}}$, as well as the gas-to-dust momentum and energy transfer rates, $\mathbf{F}_{\mathrm{gd}}$ and $\mathrm{Q}_{\mathrm{gd}}$, is given by Körösmezey and Gombosi (1989). Both multidimensional dusty gasdynamic inner coma models include a simplified external heating due to the photodissociation of water molecules (Kitamura, 1986; Körösmezey and Gombosi, 1989). 


\subsection{NUMERICAL TECHNIQUES AND BOUNDARY CONDITIONS}

In Kitamura's calculations (Kitamura, 1986, 1987), the outflowing gas temperature at the surface was taken to be $200 \mathrm{~K}$ and the initial radial expansion velocity was assumed to be the local sound velocity, i.e., $u($ outflow) $=350 \mathrm{~m} / \mathrm{s}$. In his first calculation (isotropic background), the outflowing gas concentration was taken to be

$$
\mathrm{n}(\Theta)=\mathrm{n}_{0}\left((\alpha-1) \exp \left[-\frac{\Theta^{2}}{\Theta_{0}^{2}}\right]+1\right)
$$

where $\Theta$ is the zenith angle, while the e-folding angle, $\Theta_{0}$, and $\alpha$ were chosen to be $10^{\circ}$ and 10, respectively. In his second paper, Kitamura (1987) also specified the outflowing gas density and temperature as a function of solar zenith angle, thus providing sufficient inner boundary conditions for the gas flow calculation.

In all of the present axisymmetric dusty jet model calculations, the external boundary was taken to be at a cometocentric distance of $100 \mathrm{~km}$. The external boundary represents a very low pressure outside medium, acting as a "vacuum cleaner." Kitamura solved the governing equations on a $40 \times 90$ mesh: in the radial direction he had 40 logarithmically spaced grid points, while the azimuthal direction was divided into 90 equal bins. The adopted numerical schemes were an explicit MacCormack method (Kitamura, 1986) and a first-order Godunov-type method (Kitamura, 1987).

Körösmezey and Gombosi (1989) solved the coupled, time-dependent, axisymmetric continuity, momentum and energy equations for the dust-gas mixture using a second-order-accurate Godunov-type scheme with dimensional splitting. This numerical scheme is a modified version of the technique developed by van Albada et al. (1982) and is especially tuned to describe the evolution of sharp shocks and discontinuities. The equations were solved on a $40 \times 40$ mesh. A linearly spaced grid structure was adopted in the azimuthal direction (extending from $0^{\circ}$ to $180^{\circ}$ ), while in the radial direction, the step size varied with cometocentric distance. In order to be able to resolve the transonic region with appropriate detail, the immediate vicinity of the nucleus (between $6 \mathrm{~km}$ and $6.4 \mathrm{~km}$ ) was divided into ten logarithmically spaced intervals. The second region (characterized by supersonic gas flow), which extended from $6.4 \mathrm{~km}$ to $100 \mathrm{~km}$, contained 30 logarithmically spaced grid points. During the calculation, variable time steps were applied in both regions, with the condition that the Courant number always had to be less than 0.9.

In this calculation, Körösmezey and Gombosi (1989) did not model the nucleuscoma interface in a self-consistent manner. It was assumed that the sublimation temperature was uniformly $\mathrm{T}_{\text {subl }}=200 \mathrm{~K}$, but the mantle thickness and its physical structure were not specified. Instead of specifying the mantle structure and solving the selfconsistent outflow problem, the authors substituted the sublimation process by a reservoir containing a stationary perfect gas at a temperature of $\mathrm{T}=\mathrm{T}_{\text {subl }}$ and a prescribed density. The gas mass density in the reservoir (assuming water vapor molecules) was approximated in the following form: 


$$
\rho(\Theta)=\rho_{0}\left(\alpha \exp \left[-\frac{\Theta^{2}}{\Theta_{0}^{2}}\right]+\max (\cos \Theta, 0.1\}\right)
$$

where $\Theta$ is the zenith angle. The first term in the parentheses describes a subsolar narrow jet (the adopted parameter values were $\alpha=75$ and $\Theta_{0}=10^{\circ}$ ), while the second term represents an anisotropic background.

\section{Axisymmetric Jet Models}

\subsection{WEAK JET WITH CONSTANT BACKGROUND}

At this time, there are two groups who have published non-spherically symmetric dusty gasdynamic models of inner cometary atmospheres (Kitamura, 1986, 1987; Körösmezey and Gombosi, 1989). The first non-spherically symmetric model of inner cometary atmospheres was published by Kitamura (1986). In this paper, Kitamura described relatively weak Giacobini-Zinner-class comets (the adopted gas production rate was about $10^{29}$ molecules/s) with a dust-to-gas mass production rate ratio of $\chi=1$. The model considered the effects of a narrow, dusty gas jet (with a half opening angle of about $\left.10^{\circ}\right)$. The contribution of this narrow jet to the total production rate was quite small $\left(\mathrm{Q}_{\text {jet }} / \mathrm{Q}_{\text {total }} \approx 0.07\right)$. It was assumed that, at the surface, the velocity of the outflowing gas was equal to the local sound velocity, while the density of the gas varied from $2.5 \times 10^{13} \mathrm{~cm}^{-3}$ at the subsolar point to a uniform background value of $2.5 \times$ $10^{12} \mathrm{~cm}^{-3}$, with an e-folding characteristic angle of $10^{\circ}[6]$. The outflowing gas temperature at the surface was chosen to be the same as the sublimation temperature, $T_{S}=200 \mathrm{~K}$. It was recently noted by Crifo [1] that the temperature of the nearly supersonically outflowing gas has to be considerably smaller than the sublimation temperature, because the internal energy of the sublimating gas is the source of both the bulk and random energies of the outflowing gas. There are other sources of heat impact-such as photo dissemination, chemical, and dust infrared-but these are not large enough to elevate the neutral gas temperature about $\mathrm{T}_{\text {nucleus. }}$. By keeping the temperature of the outflowing gas at the sublimation temperature, Kitamura (1986) overestimated the energy and momentum available for the gas and dust acceleration.

Figure 1 shows steady-state dust density distributions obtained with Kitamura's first model (Kitamura, 1986). Inspection of Figure 1 reveals that the originally $10^{\circ}$-wide active region eventually produces a much broader jet (the effective half opening angle is about $35^{\circ}$ ). The physical reason for this broadening is that the horizontal pressure gradient generates a lateral gas flow, which, in turn, accelerates the dust grains in the horizontal direction, too. This process in effect "sweeps out" most of the grains from the subsolar region, i.e., from the region above the active region. The horizontal expansion represents an additional expansion direction for the gas released from the active region; therefore, the pressure of this gas component decreases faster than that of the background gas. Kitamura concluded that at some point, a pressure balance is reached between the background gas and the jet ejecta and this balance eventually stops the horizontal gas expansion. This means that there will be a dust accumulation near the pressure balance surface, which, in 


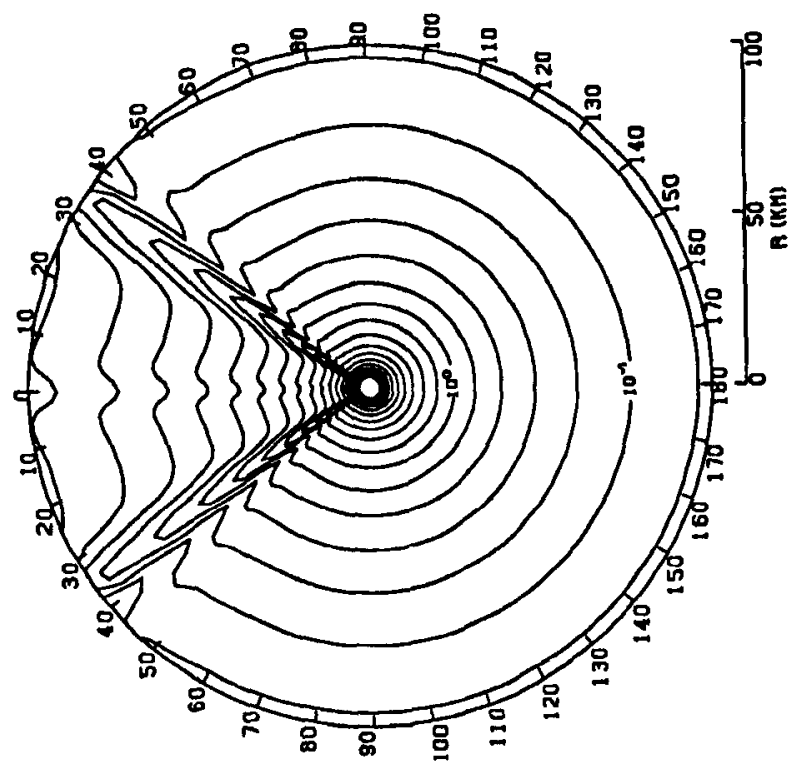

Figure 1. Dust isodensity contours in the inner cometary coma. The nucleus is modeled as an isotropic background source of gas and dust with a narrow subsolar active region. (Taken from Kitamura, 1986.) this case, happens to be a conical surface with a half opening angle of about $35^{\circ}$. Kitamura's result is very interesting: it indicates that weak jets may eventually cause a dust density decrease above the active area and that the dust accumulation is concentrated on a conical surface much beyond the angular extent of the jet itself.

\subsection{ISOLATED JETS}

In a follow-up paper, Kitamura investigated the case of isolated jets, i.e., when all dust production is concentrated to a localized subsolar active area (Kitamura, 1987). In this paper, the governing equations were solved only for the dayside (with a lowpressure free outflow

boundary at $\Theta=90^{\circ}$ ), because the fluid flow equations were not valid in the nightside region, where the gas density was very low. Inside the active area, the gas production was assumed to be isotropic with a dust-to-gas mass production rate ratio of $\chi=0.2$, while no dust was produced outside of the jet. Two different scenarios were considered in this paper. In the first case, a narrow, dusty gas jet was considered with a uniform gas production background on the sunlit hemisphere. It should be noted that this assumption resulted in a very low overall dust/gas mass ratio. It is obvious from the description of the physical problem that, in this case, azimuthal gas pressure gradients will develop only as a result of the dust loading; therefore, one can only expect moderate azimuthal gas and dust transport. Inspection of the appropriate figures in Kitamura's paper (1987) shows that this is really the case. One could say that this solution is essentially a combination of a spherically symmetric radial dusty gas flow in the subsolar region and a pure spherically symmetric radial gas flow elsewhere.

Kitamura's second dusty gas jet scenario considered a Halley-class comet $\left(\mathrm{Q} \approx 10^{30}\right.$ molecules $\left./ \mathrm{s}\right)$ with a narrow, isolated subsolar jet. In this case, all gas and dust production was confined to a narrow subsolar jet with an opening angle of $10^{\circ}$. Figure 2 shows the resulting gas and dust flow fields for dust grains with a radius of $0.01 \mu \mathrm{m}$. There is no gas production outside the jet; therefore, the radial and horizontal pressure gradients are comparable: the result is an almost exclusively azimuthal flow along the surface originating at the edge of the jet. Inside the jet, the horizontal pressure gradient is relatively 

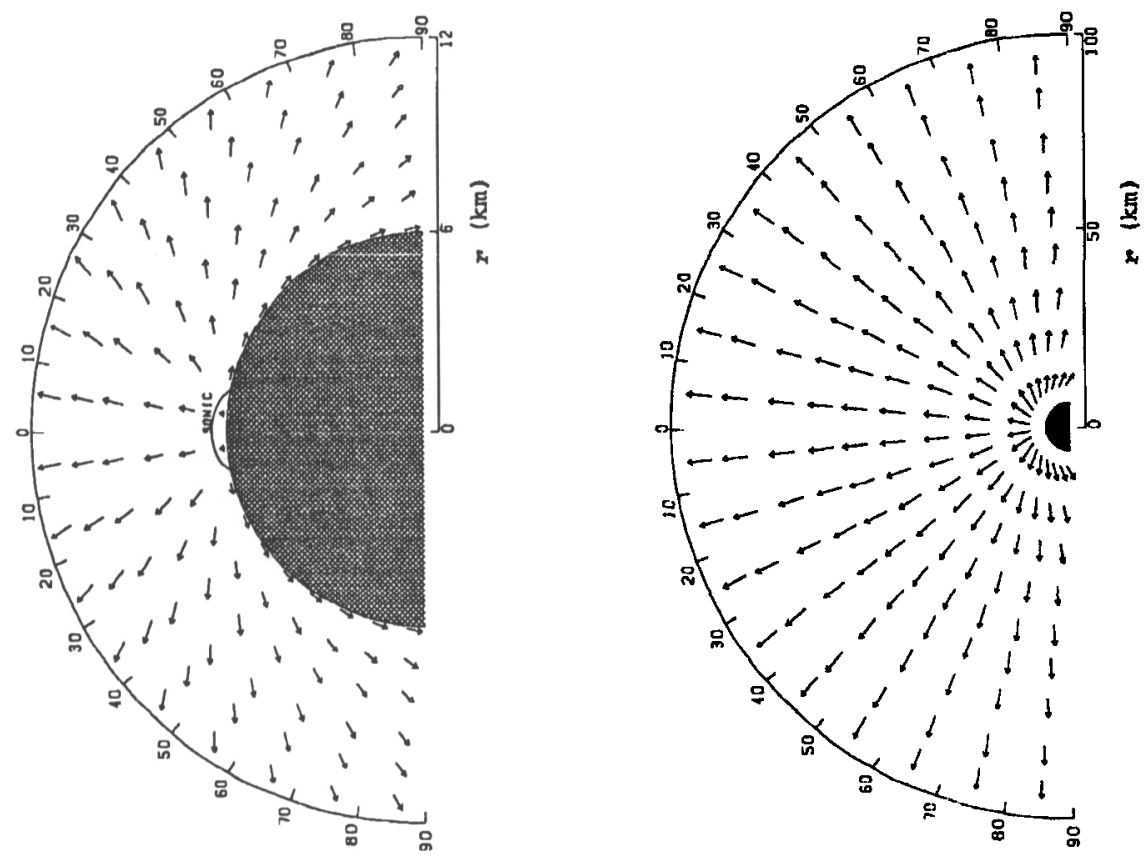

Figure 2. Gas (left panel) and $0.01-\mu \mathrm{m}$ dust (right panel) velocity fields for the case of an isolated dusty gas jet. Note that the gas velocity field is shown only up to $2 R_{\mathrm{n}}$. (Taken from Kitamura, 1987.)

small; therefore, here the outflow is nearly radial. It is interesting to note that by about $2 \mathrm{R}_{\mathrm{n}}$, the flow attains comparable radial and horizontal velocities even at the terminator. The real surprise is the dust velocity flow field. Inspection of Figure 2 reveals that even though there are considerable azimuthal velocity components in the innermost coma (within about 3 comet radii), the velocity of these small dust grains becomes practically radial beyond a few $R_{n}$.

\subsection{STRONG JET WITH ANISOTROPIC BACKGROUND}

Recently, Körösmezey and Gombosi (1989) considered several new scenarios. In one of the most interesting cases, they considered the anisotropic gas and dust production of a Halley-class comet. Without the jet, the background production rate was about $5 \times 10^{29}$ molecules/s, with a dust-to-gas mass production rate ratio of 0.3 . The background production rate varied as approximately $\mathrm{z}_{0} \cos \Theta\left(\mathrm{z}_{0}\right.$ being the subsolar production rate) for $\cos \Theta>0.1$, and was $0.1 \mathrm{z}_{0}$ for larger zenith angles. This situation, in effect, described a very broad subsolar jet with isotropic background. Figure 3 shows the steadystate gas pressure and dust mass density contours for this anisotropic background case.

The expansion of cometary gas is supported by converting the random energy of the gas molecules into organized translational motion. At the same time, the conservation 
of energy results in a rapid cooling of the expanding gas. It should be noted that, near the surface, the gas pressure gradient is much larger in the radial than in the azimuthal direction. The radial velocity of the outflowing gas at the nucleus is around $200 \mathrm{~m} / \mathrm{s}$, somewhat smaller than the local speed of sound. The large radial pressure drop results in a hypersonic radial expansion with increasing velocity. The azimuthal pressure gradient is much smaller than the radial one, but it is strong enough to generate a lateral shock propagating in the azimuthal direction with a velocity exceeding the local speed of sound. Later, this laterally propagating shock significantly weakens and it continues its azimuthal expansion as a disturbance propagating with slightly supersonic velocities. In the inner region $(r<$ $3 R_{n}$ ), the gas temperature is between about $100 \mathrm{~K}$ and approximately $150 \mathrm{~K}$; therefore, the local sound speed in water vapor is around $250 \mathrm{~m} / \mathrm{s}$. This sound speed is comparable to the velocity of radial expansion. In this near-nucleus region, lateral expansion of the jet ejecta is significant: the initial subsolar pressure peak gradually becomes a broadening conical region with a gradually increasing opening angle. Beyond about $3 R_{n}$, the gas temperature decreases to the neighborhood of $10 \mathrm{~K}$; consequently, the local sound speed drops to about $75 \mathrm{~m} / \mathrm{s}$, while the radial gas velocity component approaches $1 \mathrm{~km} / \mathrm{s}$. The azimuthal expansion velocity is approximately the same as the local sound speed, which is now about an order of magnitude smaller than the velocity of radial gas expansion; therefore, the gas flow becomes nearly radial. In this region, the expansion and broadening of the pressure peak ring becomes almost unnoticeable. In this model, all dust particles are of submicrometer size $(a=0.65 \mu \mathrm{m})$; therefore, the flow pattern of dust particles rapidly accommodates to the changing gas flow. The result is that the azimuthal flow of dust particles also becomes unimportant beyond about $3 R_{n}$. The initial rapid azimuthal expansion, followed by a gradually slowing horizontal dusty gas flow, eventually generates a nighttime dust accumulation cone, seen in Figure 3. 

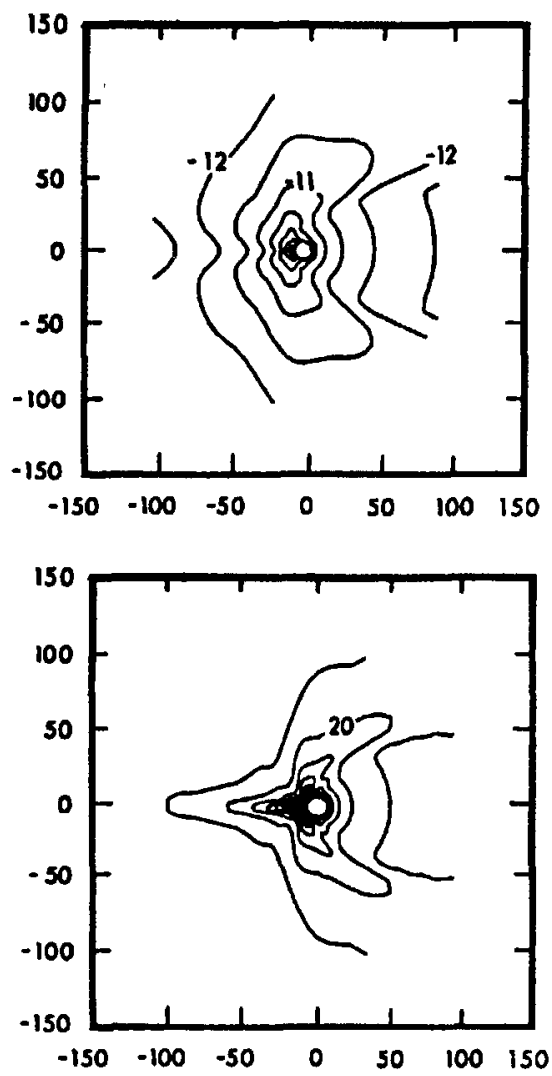

Figure 4. Gas density (left panel) and temperature (right panel) contours in the case of a strong subsolar jet with an anisotropic background. The axis of symmetry is the horizontal line going through the center. The Sun is to the left of the panel. All distances are given in kilometers.

(Taken from Körösmezey and Gombosi, 1989.)
Körösmezey and Gombosi (1989) also considered a strong, narrow jet superimposed on the anisotropic background. The source region of the jet was a narrow region around the subsolar point: the total gas and dust production coming from the jet was about 1.9 times that of the background, resulting in a total gas production rate of $1.4 \times 10^{30}$ molecules/s. The production rate distribution inside the active region had a Gaussian shape with a characteristic angular extent of $10^{\circ}$.

Figure 4 shows steady-state gas density and temperature distributions for a strong jet with an anisotropic background (Körösmezey and Gombosi, 1989). The most striking feature of the results is the appearance of a subsolar temperature spike and gas density bite-out. Also, the nighttime accumulation cone became much more pronounced. The surprising subsolar feature can be understood in terms of the gas-dust interaction inside the jet. Just above the surface (in the very first spatial cell), the dust velocity is still very small; therefore, a large number of dust particles are present. These dust particles still "remember" the surface temperature, because they did not have enough time to reach a new equilibrium temperature. It is assumed that when these hot grains interact with the gas molecules in a free molecular manner, the reflected gas molecules fully accommodate to the grain temperature. This process represents a considerable extra source of gas energy inside the active region, where the dust production rate is large. As a consequence of this interaction, the outflowing gas temperature is a strong function of the solar zenith angle. For instance, in the case of the strong jet, the temperature of the outflowing gas just above the subsolar point is about $360 \mathrm{~K}$ (the sublimation temperature is $200 \mathrm{~K}$ and the surface dust temperature is $418 \mathrm{~K}$ ), while at the antisolar point, where there is no direct sunlight and the gas production rate reaches its minimum value, the outflowing gas temperature is only about $130 \mathrm{~K}$. The increased gas temperature results in higher radial velocities (due to adiabatic conversion) and thus reduces the subsolar gas density. This effect can be clearly seen in Figure 4. 


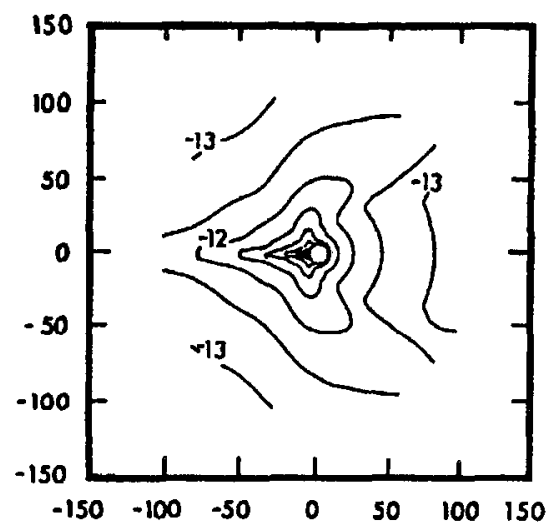

Figure 5. Dust mass density contours in the case of a strong subsolar jet with an anisotropic background. The axis of symmetry is the horizontal line going through the center. The sun is to the left of the panel. All distances are given in kilometers. (Taken from Körösmezey and Gombosi, 1989.)
Figure 5 shows the dust mass density distribution for a strong jet with an anisotropic background. Inspection of Figure 5 reveals a narrow subsolar dust density spike along the Sun-comet line. This spike is formed because the gas has no azimuthal velocity component along the axis of symmetry (the Sun-comet line); therefore, the dust cannot be swept away from this region. On the other hand, the horizontal gas velocity is quite significant near the edges of the active area; therefore, in these areas, the dust density becomes depleted, thus enhancing the relative strength of the subsolar dust spike.

Another interesting feature of the strong jet solution is the formation of the very pronounced nightside jet cone. It can be seen from Figure 5 that a significant amount of the total dust population is accumulated in this cone, in good agreement with observations (Keller et al., 1987).

\section{Nighttime Dust Accumulation}

The axisymmetric calculations of Kitamura $(1986,1987)$ and Körösmezey and Gombosi (1989) show that dust accumulation cones might be formed at large zenith angles, even when the jet itself originates from a very localized subsolar active region. In the case of weak comets (the total gas production rate is less than about $10^{29}$ molecules/s), the relatively modest azimuthal pressure gradient generates only a lateral expansion wave (and not a fast blast wave) propagating in the azimuthal direction, with a velocity near the local speed of sound. In this case, there is no initial fast azimuthal expansion and consequently the jet cone is formed on the dayside. For instance, in Kitamura's weak jet with an isotropic background, the jet cone was formed at about $50^{\circ}$, as can be seen in Figure 1 (Kitamura, 1986).

On the other hand, when an active comet was considered with anisotropic production, the jet cone was pushed back to the nightside, because the larger horizontal pressure gradient created a lateral shock, which greatly increased the nightward dust transport. The presence of a subsolar narrow jet only further enhanced this effect, increasing the amount of dust on the nightside.

In summary, axisymmetric dusty gas models suggest that active comets have nightside dust cones, even when all the dust is released on the dayside. At the same time, such a phenomenon is not predicted for less active comets.

The comet Halley images taken by the Giotto spacecraft revealed a nighttime dust component at cometocentric distances $>30 \mathrm{~km}$ from the nucleus (Keller et al., 1987; 
H.U. Keller, private communication, 1989). This dust population was interpreted by the Giotto imaging team as dust grains originating from the active areas on the dayside and swept across the terminator by the expanding gas (H.U. Keller, private communication, 1989). The results of Körösmezey and Gombosi (1989) support this idea.

\section{Acknowledgments}

This work was supported by NSF grant AST-8605994, and NASA grant NAGW1366. Acknowledgement is also made to the National Center for Atmospheric Research sponsored by NSF, for the computing time used in this research.

\section{References}

1. Crifo, J.F. (1988) 'Collisional coma models: An unorthodox overview,' Adv. Space Res., in press.

2. Crifo, J.F. (1989) 'Inferences concerning water vapour viscosity and mean free path at low temperatures,' Astron. Astrophys., in press.

3. Gombosi, T.I., and Körösmezey, A. (1988) 'Modeling of the cometary nucleus coma interface region,' Adv. Space Res., in press.

4. Gombosi, T.I., and Körösmezey, A. (1989) 'Cometary dusty gas dynamics,' in Outstanding Problems in Solar System Physics, edited by J. Burch and J.H. Waite, in press.

5. Keller, H.U., Delamere, W.A., Huebner, W.F., et al. (1987) 'Comet P/Halley's nucleus and its activity,' Astron. Astrophys., 187, 807.

6. Kitamura, Y. (1986) 'Axisymmetric dusty gas jet in the inner coma of a comet,' Icarus, 66, 241.

7. Kitamura, Y. (1987) 'Axisymmetric dusty gas jet in the inner coma of a comet II. The case of isolated jets,' Icarus, 72,555 .

8. Körösmezey, A., and Gombosi, T.I. (1989) 'A time dependent dusty-gas dynamic model of axisymmetric cometary jets,' Icarus, in press.

9. Probstein, R.F. (1968) 'The dusty gas dynamics of comet heads,' in Problems of Hydrodynamics and Continuum Mechanics, Society of Industrial Application Mathematics, p. 568.

10. van Albada, G.D., van Leer, B., and Roberts, W.W. (1982) 'A comparative study of computational methods in gas dynamics,' Astron. Astrophys., 108, 76. 